\title{
THE MECHANISM OF POLLEN INCOMPATIBILITY IN THEOBROMA CACAO L.
}

\author{
F. W. COPE \\ Regional Research Centre, University College of the West Indies, \\ Imperial College of Tropical Agriculture, Trinidad
}

Received 7.viii.6r

\section{INTRODUCTION}

INCOMPATIBILITY has been defined by Mather (1943) as " the failure, following mating or pollination, of a male gamete and a female gamete to achieve fertilization, where each of them is capable of uniting with other gametes of the breeding group after similar mating or pollination". This definition appears to be particularly appropriate to the phenomena associated with fertilization in Theobroma cacao L., for it will be shown in this paper that the abscission of incompatibly pollinated flowers of that species is intimately connected with the regular presence, in the pollinated ovary, of ovules wherein male and female gametic nuclei have failed to fuse and (usually) of other ovules wherein syngamy has taken place. In the great majority of compatibly pollinated ovaries, on the other hand, ovules showing fusion failure are completely absent.

Knight and Rogers (1953, I955) concluded, from the results of numerous hand pollinations, that $\mathcal{T}$. cacao belongs to the group of flowering plants in which the incompatibility reaction is known to be sporophytically determined, the diploid constitutions of both male and female parents being involved in determining the compatibility or incompatibility of a cross-pollination. They postulated the existence, in their three SI parent trees, of a series of five * alleles, at a single locus, which showed dominance and independence relationships, alike in male and female parts of the flower.

The site of the incompatibility reaction in cacao lies not in the style but in the embryo sac. The reaction, indeed, is not determined until the male gametes, delivered into the embryo sac, come to lie in contact with their female counterparts. Where the pollination is incompatible, the proportion of "non-fusion" ovules in the ovary is either 25 per cent., 50 per cent. or nearly, if not quite roo per cent. of all ovules receiving male gametes ("fertilized ovules").

T. cacao provides the first example, amongst the flowering plants, of an incompatibility mechanism based on genetic control of the success or failure of syngamy.

\section{PREVIOUS INVESTIGATIONS ON T. CACAO}

Pound (1932) was the first to discover the incidence of self- and cross-incompatibility in cacao. He showed also that the Trinidad SI tree required pollen from the $\mathrm{SC}$ tree before it could fruit. Marshall

* From investigations reported in this paper, it would seem more likely that six alleles, and not five, exist in the parent material. 
(1933), Posnette (1937), Voelcker (1937, I938) and Cope (1939a, b), all working in Trinidad, confirmed Pound's early findings. Incompatibility has not, however, manifested itself only in Trinidad. Ostendorf (1948) found evidence of it in Java cacao. Müntzing (1947) successfully crossed two SI cacao trees in Ecuador. Naundorf and Villamil (1950) recognised the incidence of incompatibility in Colombian cacao. All members of a sample from a population of trees introduced by Pound (1938) into Trinidad from the upper reaches of the Amazon were found by Posnette (1945) to be SI and, to a limited degree, cross-compatible; these trees readily crossed with SI trees of Trinidad origin. Cope (unpublished) found cacao trees in Grenada to be either SI or SC, and a small group of trees growing wild on the Rio Apoporis in Colombia was found by him to contain only SI members, between which cross-compatibility obviously existed.

Cytological investigations on incompatibility were begun by Cope (1939b, 1940). From stained squash-mounts of entire styles and from microtomed sections of ovaries he concluded that in incompatible matings the pollen tubes grow no less rapidly and no less directly down the style than do compatible tubes, and that incompatible tubes penetrate and deliver gametes into the ovules. These investigations were resumed in 1948 and the results appear in this paper.

The genetic system controlling incompatibility in cacao was studied by Knight and Rogers (1953, 1955). They investigated patterns of flower abscission and retention in cross-pollinations within and between three groups of progenies derived from crosses between three SI parents of upper Amazon origin. Every individual progeny was SI. Within each progeny group either three or four intraincompatible and inter-compatible sub-groups could be distinguished. Their genetic hypothesis, which is identical with Scheme J of Bateman (1952), has been outlined above.

\section{MATERIALS AND METHODS}

The materials used were of diverse origins. The Trinitario trees studied belong to the 100 clones selected by Pound in the early 1930's from the cacao population of 'Trinidad and Tobago, and forming the ICS series. Two of these, ICS 45 and ICS 6o, are probably recent hybrids between Central American cacaos and local material. The Amazon material studied came from Pound's collections in the Amazon valley. The author has been fortunate in being able to study, on the spot, some of the trees used by Knight and Rogers. Three clones from Ecuador were included in the investigations. Numerous seedling trees, the progenies of crosses and selfings made under a plant breeding programme, have been individually examined for their reactions to self- and sib-pollination and to back-pollination to both parents.

Flowers used for studies were always guarded from insect visits. When open, the flower was either selfed, or cross-pollinated from a similarly guarded flower, and the guard tube replaced for another 24 hours. No emasculation was practised because the peculiar structure of the small, hanging flower tends to prevent the dislodgment of pollen from the anthers, which project into cup-shaped petal bases. In early studies flowers were collected at $24,48,72$ and 96 hours after pollination; in later studies flowers were collected only at 72 hours. After a light paring of its 
wall, the ovary was fixed for $\mathbf{2 4}$ hours in Io ml. of Craf fixative (Randolph, 1935). The material was then taken up to paraffin wax through a series of ethyl-tertiary butyl alcohol mixtures (Johansen, 1940) and sectioned at i5 $\mu$. Staining was carried out with iron-hæmatoxylin (Haidenhain's), the sections being differentiated in a saturated aqueous solution of picric acid allowed to act for 4-6 minutes.

The slides were microscopically examined under a magnification of goo times, each loculus being followed through until all the ovules in the ovary had been examined. Note was made, for each ovule, whether or not one synergid (or both) had collapsed, whether or not one male gamete had fused with the egg nucleus, and whether or not the second male gamete had fused with the polar nuclei. Some 90,000 ovules were examined in all.

The data recorded for individual ovaries consist of two parts, the number $(p)$ of ovules showing non-fusion of gametes, and the number $(q)$ showing normal fusion and post-fertilization development. The ratio of $p$ to $p+q$ (denoted by $\mathbf{P}$ ) varies, of course, from one ovary to the next ; for unavoidable reasons, $p+q$ (denoted by $n$ ) also varies between observations. In order to allow analyses of variance to be made on the (usually) replicated observations, some normalizing transformation function was sought which would allow the variances of the transformed $P$ values to become additive. One such transformation (Yates, 1953), the half-logit, defined as $z=\frac{1}{2}(\ln \mathrm{Q}-\ln \mathrm{P})$, where $\mathrm{Q}=\mathrm{I}-\mathrm{P}$, was used. The individual transforms, which all possess different accuracies because of the variation in $n$, were weighted. The variance of $z$ is $\mathrm{I} / 4 n \mathrm{PQ}$, so that the most appropriate weight for each observation was considered to be $4 n \mathrm{PQ}$, or $4 p q / n$. Apart from analyses of within- and betweentree variances, and the computation of means and associated standard errors for sundry groups of data, $x^{2}$ analyses of the deviation of the individual $p / q$ ratios from their overall ratio were made to test the homogeneity of the ratio. The contingency- $x^{2}$ values were then distributed amongst the classes bounded by values of $x^{2}$ for one degree of freedom, and the frequencics compared with expectation. The frequency distribution of non-fusion ovules in groups of ovules defined by the loculi was also analysed, using the expansion of $[\Sigma p / \Sigma(p+q)+\Sigma q / \Sigma(p+q)]^{r}$ to calculate the expected frequencies, where $r$ is the number of ovules per loculus. A $x^{2}$ test of the goodness of fit between actual and expected frequencies was made for the purpose of testing the hypothesis that encounters between non-fusing gametes were the result of purely random processes.

\section{RESULTS}

Self-pollinations, and compatible pollinations with pollen of ICS I, were made on trees of ICS 43 and ICS 53, two clones long known not to set fruit from selfing. In the selfed series of ovaries, it was noted that at the time when the flowers absciss-usually three or four days after pollination-a proportion of the ovules shows one male gamete not to have fused with the egg nucleus (plate, figs. I and 3) and the second male gamete, likewise, not to have fused with the polar nuclei (plate, figs. 2 and 3 ). In the case of the egg nucleus, the male gamete invariably lies in apparent contact with it, both retaining their spherical shape. The second male gamete, however, quite frequently lies detached from the polar nuclei; or the male gamete is associated with one polar nucleus, the other having separated from its partner. Dissociated polars occasionally undergo mitosis, and in this process the second male gamete, very rarely, may join. Polar nuclei in non-fusion ovules are characterised by poor staining properties; their nucleoli remain unenlarged and the nucleoplasm 
appears thin and scanty. The other fertilized ovules in these incompatibly selfed ovaries show one male gamete to fuse with the egg nucleus to give a prominently bi-nucleolate zygote, and the other to fuse with the polar nuclei, which never separate. The triploid endosperm nucleus (plate, fig. 4) appears capable of almost immediate division.

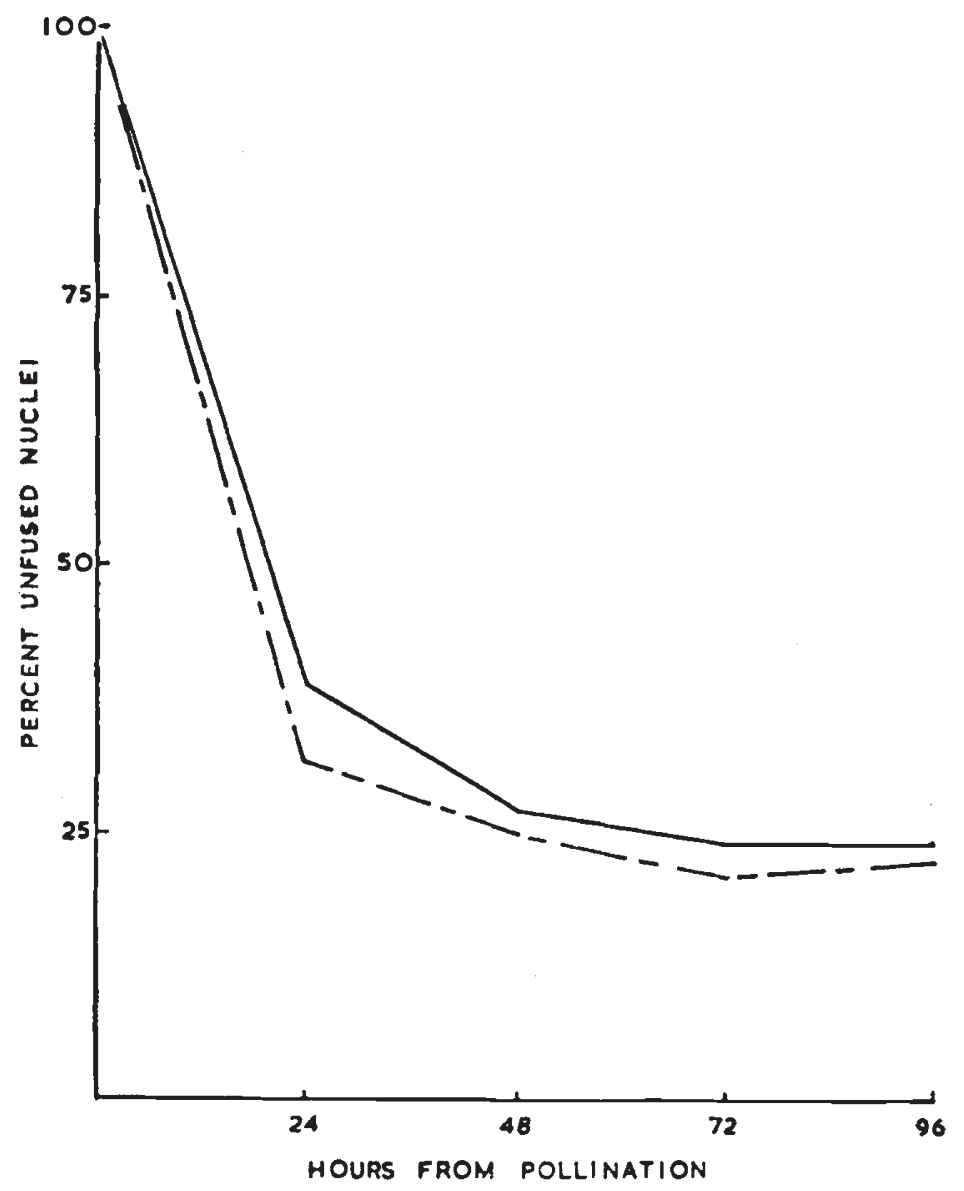

TEXT-FIG. I.

The progress of syngamy is shown graphically in text-fig. I for incompatible self-pollinations, and in text-fig. 2 for compatible crosspollinations. Text-fig. I shows that relatively little change in the proportion of ovules with unfused gametes takes place after 48 hours from pollination, the curve flattening rapidly to a final 25 per cent. level at 72 hours. Text-fig. 2 shows, on the other hand, that the proportion of ovules in which fusion has not occurred decreases without interruption, in compatibly pollinated ovaries, until at 96 hours from pollination all fertilized ovules show syngamy to have taken place (though very occasionally an ovule with unfused gametes may be 
seen). It seems highly unlikely, therefore, that the presence of numerous non-fusion ovules in incompatibly pollinated ovaries at the time of abscission is the result of late delivery of male gametes into the embryo-sac, and improbable that, given time, these gametes would fuse with their female counterparts.

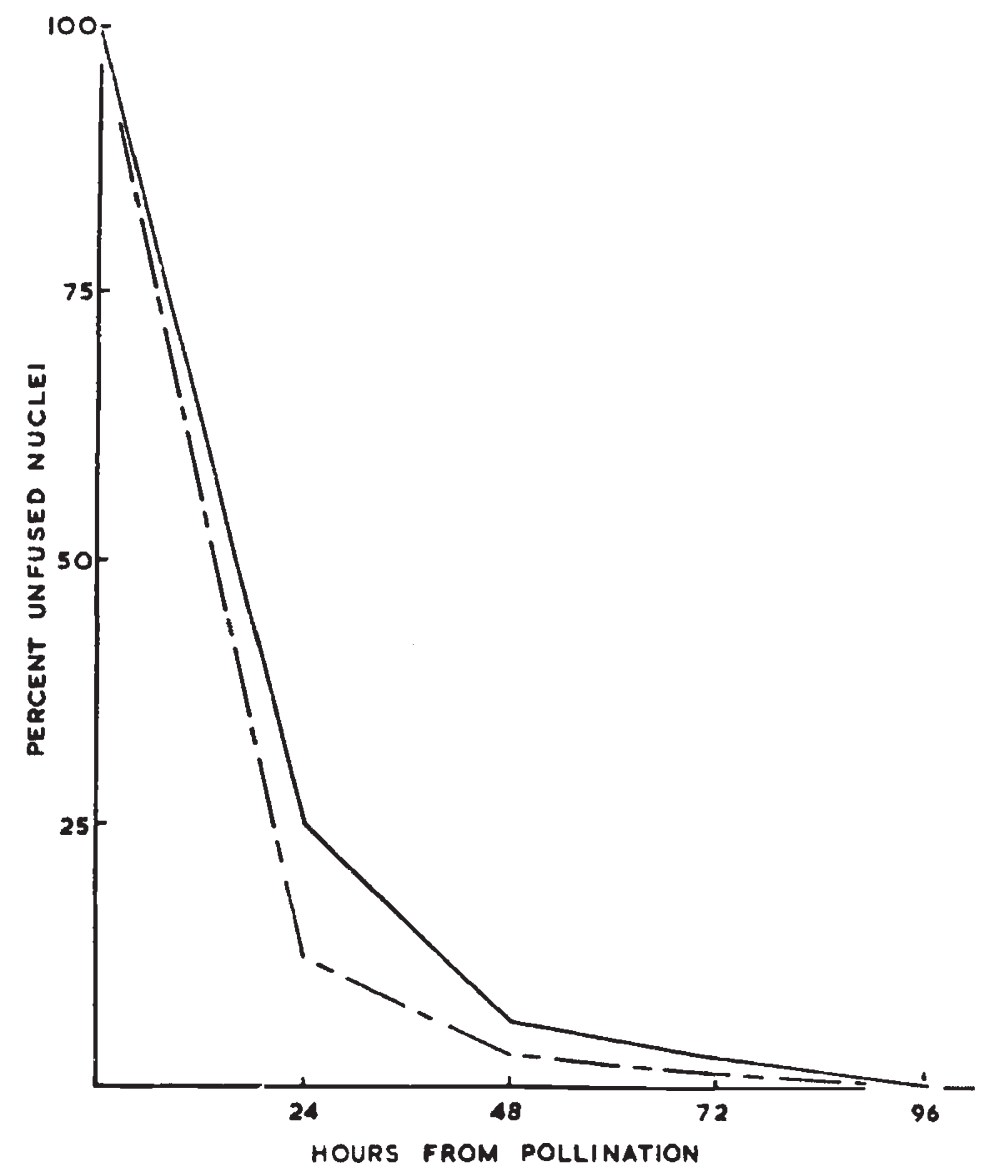

TEXT-FIG. 2.

Text-Figs. I and 2.--Progress of nuclear fusion in embryo-sacs of ICS 43 and ICS 53 in relation to time after incompatible self-pollination (text-fig. I) and compatible crosspollination (text-fig. 2). Solid line for egg and male gamete fusions, pecked line for polar nuclei and male gamete fusions.

The post-fertilization nuclear content of embryo-sacs of the normal ovules in incompatibly pollinated ovaries is microscopically identical with that of embryo-sacs in compatibly crossed ovaries. The similarity extends to nuclear activity. Text-fig. 3 shows that the proportion of ovules in which the first division of the triploid polar nucleus has occurred is very similar, in relation to time, in both cases. Mitotic activity in other parts of the ovule, however, becomes markedly different in the two groups after the 48 -hour stage has been reached. 
When the incompatibly pollinated flower abscisses, the normal ovules present in its ovary show much reduced levels of mitotic activity in both nucellus and integuments; activity in these tissues is less than half that in compatibly pollinated ovaries at 72 hours after pollination, and only one-sixth at 96 hours (text-fig. 4). Even more marked is the decline in mitotic activity of the nucellus and integuments in

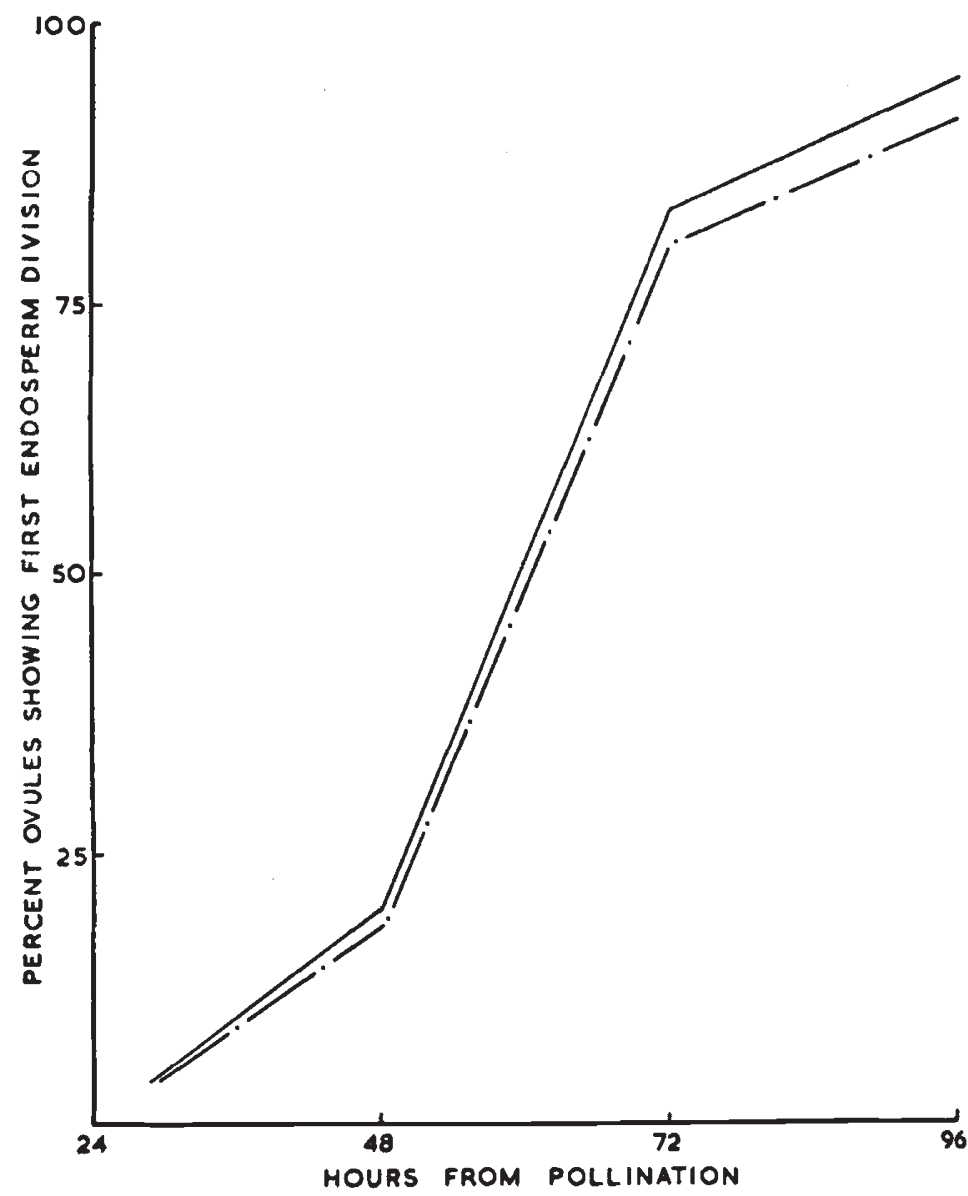

TEXT-FIG. 3.-Percentage of fertilized ovules showing first division of the primary endosperm body in relation to time after pollination. Solid line for compatible crosspollinations on ICS 43 and ICS 53 , pecked line for incompatible self-pollinations.

the non-fusion ovules in incompatible matings, for here such activity

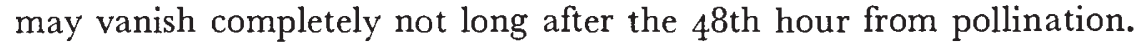

Non-fusion ovules can usually readily be distinguished under lowpower magnification in sections of ovaries fixed 72 hours after pollination, for their staining is much less intense than the normal ovules which accompany them. Under higher magnification the nucellar and integumental cells are seen to possess very poor cytoplasmic contents, and the nuclei appear smaller and less regularly spherical than those in tissues belonging to normal ovules. The nucellar cells 
immediately adjoining the embryo-sac frequently appear spindleshaped; their heavy absorption of stain may indicate some form of protoplasmic breakdown.

During the examination of sectioned ovaries from guarded and unpollinated flowers of ICS 43 and ICS 53, some observations were

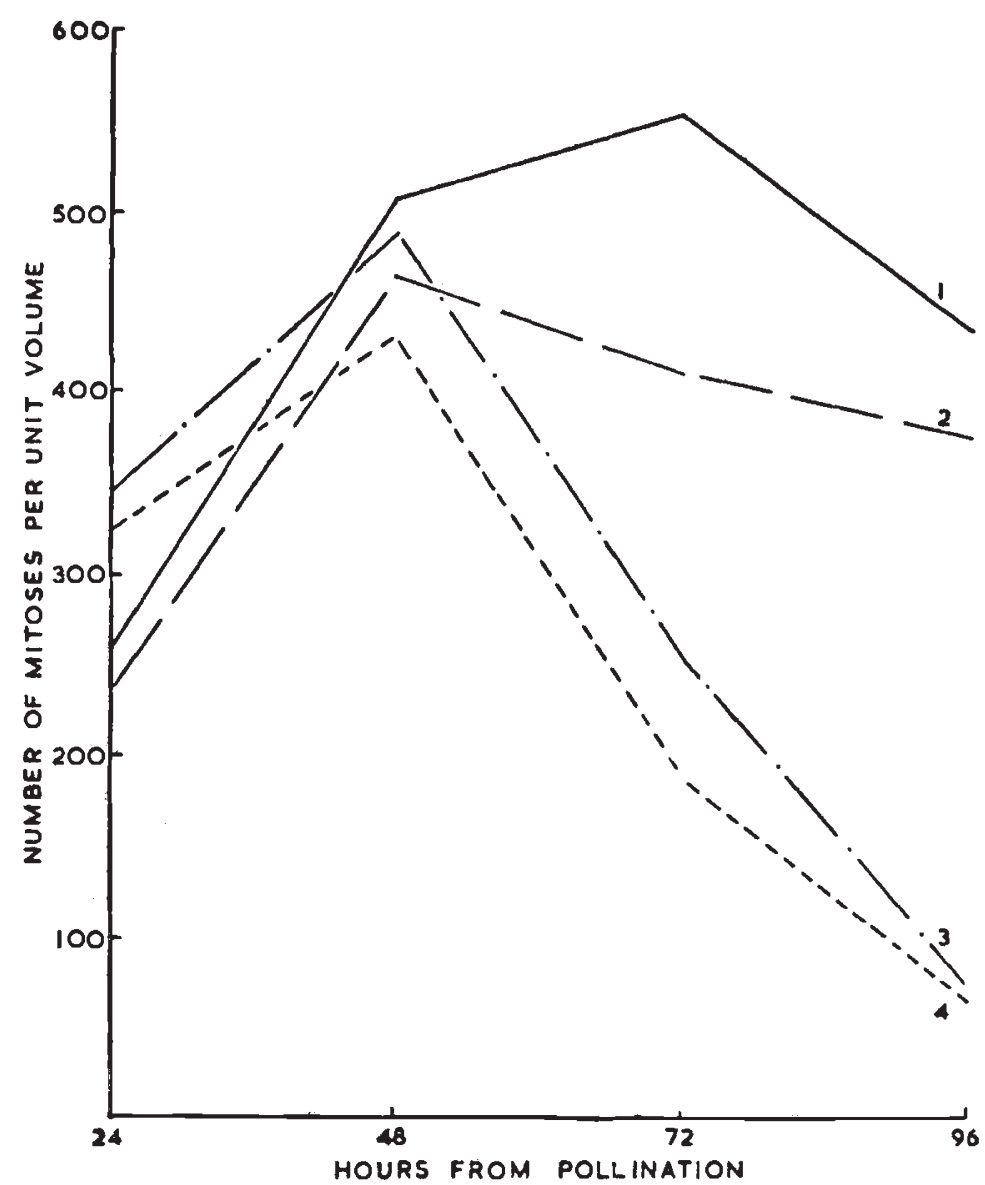

TEXT-FIG. 4.-Mitotic activity in nucellus and integuments, in relation to time after pollination, in compatibly cross-fertilized and incompatibly self-fertilized ovules of ICS 43 and ICS 53 .
(I) Integuments, compatibly crossed
(2) Nucellus, compatibly crossed
(3) Integuments, incompatibly selfed
(4) Nucellus, incompatibly selfed.

made to the effect that in the absence of pollen tube entry into the embryo-sac, the egg nucleus occasionally fuses with one of the synergid nuclei. Where this occurs in an ovule, one synergid collapses and absorbs stain heavily (its nucleus presumably having migrated to the egg cell), the egg nucleus becomes bi-nucleolate and much enlarged, whilst the polar nuclei retain the appearance they have in unfertilized ovules. In other studies, with pollinated flowers, similar conformations 
were encountered; one case was seen where the egg, migrated synergid nucleus and a male nucleus were all together in contact in the egg cell. Ovules in which a synergid nucleus fuses with the egg nucleus appear, however, quite incapable of further development, undoubtedly due to the non-fertilization of the polar nuclei. The observations are of interest in suggesting that female nuclei of identical genetic constitution experience no barrier to fusion, such as is the case between male and female nuclei in certain circumstances.

Both ICS 43 and ICS 53 gave the same 25 per cent. incidence of non-fusion ovules in selfed ovaries. The similarity suggested that the proportion was of fundamental significance in the incompatibility reaction. The scope of the investigations was therefore extended to embrace, as widely as possible, known cases of self- and cross-incompatibility. Fifty clones of the ICS series with records of non-setting from self-pollinations were chosen for study, and 14 of them were randomly selected for cross-pollination studies. The material used by Posnette (1945) in his studies establishing the existence of crosscompatibility between SI trees was also available. In these and all subsequent investigations ovaries at the 72 -hour stage only were taken for sectioning.

Investigations on the selfing of the 50 ICS SI clones completely confirmed the results from ICS 43 and ICS 53 . Triplicate selfings on each clone yielded a total of 1434 non-fusion (25.12 per cent.) and 4275 normal ovules (table 1). The between-tree variance of $0.716 \mathrm{I}$, calculated from the transformed counts, does not differ significantly from the within-tree variance of 0.8327 . Table 2 shows the frequencies with which the 150 contingency- $\chi^{2}$ values fall into magnitude classes and the expected frequencies; the goodness-of-fit $\chi^{2}$ value obtained would be equalled or exceeded in some 40 per cent. of similar trials. The counts are therefore statistically homogeneous, the amount of "spread" in the $p / q$ ratio, from ovary to ovary, being no larger or smaller than the effects of random sampling would require. In table 3 appear the actual and expected frequencies with which loculi containing from five to ten $(n)$ fertilized ovules included from zero to $n$ non-fusion ovules. The fit is good, a fact which suggests that each ovule becomes a non-fusion or a normal ovule independently of the others in the same loculus.

The study of cross-pollinations made in all possible combinations on the I4 mutually cross-incompatible SI ICS clones yielded totals of $180 \mathrm{I}(25.29$ per cent.) non-fusion and $532 \mathrm{I}$ normal ovules from 182 duplicate ovaries. Between- and within-tree variances did not differ significantly, and the counts showed homogeneity. Compatible cross-pollinations of these I4 clones with two SI Amazon clones, PA 7 and NA 32, gave 1372 fertilized ovules for examination; not one was of the non-fusion type.

Self- and cross-pollinations made by Posnette (1945) on I I trees belonging to three distinct populations of Amazon cacao (Nanay, 
Parinari and Iquitos) were repeated. Two other upper Amazon clones, SCA 6 and SCA 12 ; three Ecuadorian clones, E $5, \mathrm{E}_{5}$ and $E_{17}$; and two Trinitario clones, ICS I and ICS 6o, were included

TABLE ,

Ovule counts in triplicate from selfed ovaries of 50 SI ICS clones

L.H. number refers to non-fusion ovules, R.H. number to normal ovules

\begin{tabular}{|c|c|c|c|c|c|c|c|c|c|c|c|c|c|c|}
\hline \multicolumn{3}{|c|}{ ICS } & \multicolumn{3}{|c|}{ ICS } & \multicolumn{3}{|l|}{ ICS } & \multicolumn{3}{|c|}{ ICS } & \multicolumn{3}{|c|}{ ICS } \\
\hline 2 & $\begin{array}{l}5 \\
5 \\
7\end{array}$ & $\begin{array}{l}19 \\
17 \\
23\end{array}$ & 19 & $\begin{array}{r}8 \\
11 \\
9\end{array}$ & $\begin{array}{l}32 \\
34 \\
3^{8}\end{array}$ & 43 & $\begin{array}{r}9 \\
8 \\
10\end{array}$ & $\begin{array}{l}34 \\
28 \\
24\end{array}$ & $5^{8}$ & $\begin{array}{l}12 \\
11 \\
16\end{array}$ & $\begin{array}{l}28 \\
27 \\
25\end{array}$ & 80 & $\begin{array}{r}11 \\
9 \\
9\end{array}$ & $\begin{array}{l}24 \\
33 \\
34\end{array}$ \\
\hline 3 & $\begin{array}{r}11 \\
11 \\
8\end{array}$ & $\begin{array}{l}27 \\
31 \\
28\end{array}$ & 22 & $\begin{array}{r}9 \\
3 \\
11\end{array}$ & $\begin{array}{l}18 \\
16 \\
19\end{array}$ & $4^{6}$ & $\begin{array}{r}6 \\
11 \\
11\end{array}$ & $\begin{array}{l}36 \\
34 \\
33\end{array}$ & 60 & $\begin{array}{l}14 \\
12 \\
10\end{array}$ & $\begin{array}{l}31 \\
25 \\
32\end{array}$ & 82 & $\begin{array}{l}10 \\
12 \\
11\end{array}$ & $\begin{array}{l}35 \\
34 \\
35\end{array}$ \\
\hline 4 & $\begin{array}{r}6 \\
14 \\
7\end{array}$ & $\begin{array}{l}24 \\
25 \\
28\end{array}$ & 26 & $\begin{array}{r}9 \\
7 \\
14\end{array}$ & $\begin{array}{l}30 \\
32 \\
25\end{array}$ & 47 & $\begin{array}{r}8 \\
10 \\
9\end{array}$ & $\begin{array}{l}32 \\
36 \\
36\end{array}$ & 64 & $\begin{array}{r}6 \\
12 \\
9\end{array}$ & $\begin{array}{l}21 \\
31 \\
28\end{array}$ & 83 & $\begin{array}{r}17 \\
7 \\
10\end{array}$ & $\begin{array}{l}25 \\
3^{0} \\
3^{1}\end{array}$ \\
\hline 5 & $\begin{array}{r}11 \\
10 \\
9\end{array}$ & $\begin{array}{l}23 \\
29 \\
3^{2}\end{array}$ & 34 & $\begin{array}{l}10 \\
12 \\
11\end{array}$ & $\begin{array}{l}32 \\
26 \\
38\end{array}$ & $4^{8}$ & $\begin{array}{r}9 \\
12 \\
11\end{array}$ & $\begin{array}{l}26 \\
25 \\
32\end{array}$ & 66 & $\begin{array}{r}10 \\
12 \\
6\end{array}$ & $\begin{array}{l}30 \\
95 \\
30\end{array}$ & 84 & $\begin{array}{l}15 \\
10 \\
10\end{array}$ & $\begin{array}{l}23 \\
30 \\
31\end{array}$ \\
\hline 11 & $\begin{array}{r}9 \\
14 \\
4\end{array}$ & $\begin{array}{l}24 \\
24 \\
27\end{array}$ & 35 & $\begin{array}{r}9 \\
8 \\
13\end{array}$ & $\begin{array}{l}28 \\
30 \\
3^{2}\end{array}$ & $5^{0}$ & $\begin{array}{l}8 \\
5 \\
6\end{array}$ & $\begin{array}{l}32 \\
28 \\
31\end{array}$ & 71 & $\begin{array}{r}9 \\
\text { ro } \\
9\end{array}$ & $\begin{array}{l}26 \\
24 \\
30\end{array}$ & 86 & $\begin{array}{l}7 \\
7 \\
9\end{array}$ & $\begin{array}{l}28 \\
32 \\
22\end{array}$ \\
\hline 13 & $\begin{array}{r}9 \\
2 \\
12\end{array}$ & $\begin{array}{l}30 \\
22 \\
3^{6}\end{array}$ & 37 & $\begin{array}{r}14 \\
9 \\
15\end{array}$ & $\begin{array}{l}26 \\
33 \\
26\end{array}$ & $5^{1}$ & $\begin{array}{l}12 \\
11 \\
13\end{array}$ & $\begin{array}{l}27 \\
29 \\
27\end{array}$ & 72 & $\begin{array}{r}7 \\
6 \\
10\end{array}$ & $\begin{array}{l}25 \\
27 \\
26\end{array}$ & 87 & $\begin{array}{r}8 \\
7 \\
12\end{array}$ & $\begin{array}{l}32 \\
36 \\
30\end{array}$ \\
\hline 15 & $\begin{array}{r}14 \\
6 \\
8\end{array}$ & $\begin{array}{l}23 \\
34 \\
24\end{array}$ & 39 & $\begin{array}{r}7 \\
11 \\
10\end{array}$ & $\begin{array}{l}28 \\
27 \\
35\end{array}$ & $5^{2}$ & $\begin{array}{l}11 \\
12 \\
12\end{array}$ & $\begin{array}{l}28 \\
28 \\
30\end{array}$ & 74 & $\begin{array}{r}5 \\
11 \\
10\end{array}$ & $\begin{array}{l}21 \\
24 \\
34\end{array}$ & 89 & $\begin{array}{l}9 \\
4 \\
3\end{array}$ & $\begin{array}{l}27 \\
29 \\
35\end{array}$ \\
\hline 36 & $\begin{array}{l}10 \\
10 \\
12\end{array}$ & $\begin{array}{l}25 \\
32 \\
36\end{array}$ & 40 & $\begin{array}{r}12 \\
11 \\
9\end{array}$ & $\begin{array}{l}24 \\
35 \\
32\end{array}$ & 53 & $\begin{array}{l}7 \\
7 \\
4\end{array}$ & $\begin{array}{l}23 \\
27 \\
35\end{array}$ & 75 & $\begin{array}{r}8 \\
10 \\
12\end{array}$ & $\begin{array}{l}31 \\
37 \\
29\end{array}$ & 92 & $\begin{array}{r}12 \\
5 \\
11\end{array}$ & $\begin{array}{l}24 \\
32 \\
37\end{array}$ \\
\hline 37 & $\begin{array}{r}7 \\
9 \\
14\end{array}$ & $\begin{array}{l}24 \\
32 \\
20\end{array}$ & $4^{1}$ & $\begin{array}{r}9 \\
6 \\
12\end{array}$ & $\begin{array}{l}30 \\
31 \\
26\end{array}$ & $5^{6}$ & $\begin{array}{l}11 \\
10 \\
13\end{array}$ & $\begin{array}{l}21 \\
26 \\
27\end{array}$ & 77 & $\begin{array}{r}11 \\
6 \\
10\end{array}$ & $\begin{array}{l}20 \\
32 \\
28\end{array}$ & 94 & $\begin{array}{r}10 \\
8 \\
9\end{array}$ & $\begin{array}{l}25 \\
30 \\
28\end{array}$ \\
\hline 18 & $\begin{array}{l}13 \\
11 \\
12\end{array}$ & $\begin{array}{l}30 \\
31 \\
33\end{array}$ & $4^{2}$ & $\begin{array}{l}12 \\
11 \\
12\end{array}$ & $\begin{array}{l}27 \\
27 \\
28\end{array}$ & 57 & $\begin{array}{r}10 \\
16 \\
7\end{array}$ & $\begin{array}{l}38 \\
34 \\
30\end{array}$ & 78 & $\begin{array}{l}8 \\
5 \\
9\end{array}$ & $\begin{array}{l}25 \\
18 \\
26\end{array}$ & 99 & $\begin{array}{r}10 \\
3 \\
10\end{array}$ & $\begin{array}{l}24 \\
22 \\
17\end{array}$ \\
\hline & & & & & & & & 1434 & 42 & & & & & \\
\hline
\end{tabular}

in the investigation. In every case of failure to set fruit reported by Posnette non-fusion ovules were present in ovaries from the same crosses; 629 non-fusion ovules $(24.25$ per cent.) appeared amongst 2594 fertilised ovules examined. In every case of reported fruit setting the ovaries contained only normal ovules; $5 \mathrm{r} 40$ ovules were examined. The two SCA clones were found to be SI and cross-incompatible, 
but they crossed readily with all other materials used in the studies. SCA 6, on selfing, gave counts from four ovaries:

$\begin{array}{cc}\begin{array}{c}\text { Non-fusion } \\ \text { ovules }\end{array} & \begin{array}{c}\text { Normal } \\ \text { ovules }\end{array} \\ 25 & 26 \\ 14 & 26 \\ 22 & 19 \\ 18 & 13 \\ - & -\overline{84} \\ 79 & \end{array}$

so that SCA 6 provided a second type of SI tree giving, when selfed, an approximate 50 per cent. content of non-fusion ovules in the ovary.

The three clones from Ecuador were assumed at the outset to be SI. Selfed ovaries of E I 5 and E I 7 were found, however, to contain only normal ovules, and the SC status of these two clones was confirmed by numerous settings from hand-pollinations. E 5, on the other hand, regularly contained some 25 per cent. of non-fusion ovules in its selfed ovaries and in those cross-pollinated with two of the four Nanay clones; these findings were checked by hand-pollinations, from which no setting ever resulted.

\section{TABLE 2}

Frequency distribution of contingency- $\chi^{2}$ values (calculated from ratio $1434: 4275$ ) for the 150 ovule counts of table $I$

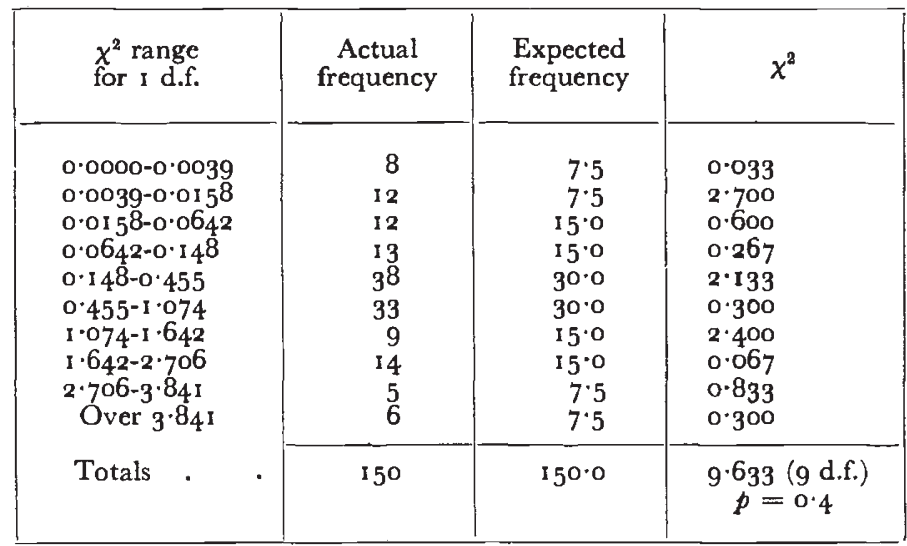

Statistical analyses of all the results in this repetition and extension of Posnette's work-excluding SCA 6 selfed-showed that (I) the within- and between-tree variances attaching to ovule counts in incompatible selfings and crosses were always similar in magnitude; (2) the actual frequency distribution of contingency- $\chi^{2}$ values fitted well with expectation; (3) the encounter of gametes unable to fuse was a randomly controlled process.

Besides the 50 SI ICS clones mentioned above, the other clones of the series received some attention. Of the Ioo clones, 99 show 
agreement between the compatibility status determined by the fall or retention of self-pollinated flowers and that determined by the presence or absence of non-fusion ovules in selfed ovaries-the one aberrant clone has almost certainly become mis-labelled in the course of repeated vegetative propagation. Thirty-six of these 99 clones are

TABLE 3

Frequency distribution of numbers of ovules ( $\mathrm{r}$ ) showing non-fusion in loculi containing 5-10 fertilized ovules $(\mathrm{n})$. Expected frequencies are based on expansion of $(0 \cdot 2512+0 \cdot 7488)^{n}$. Based on the 150 counts of table I

\begin{tabular}{|c|c|c|c|c|c|c|c|c|c|c|c|c|}
\hline \multirow{3}{*}{$r$} & \multicolumn{12}{|c|}{ Number of fertilized ovules in loculus $(n)$} \\
\hline & \multicolumn{2}{|c|}{5} & \multicolumn{2}{|r|}{6} & \multicolumn{2}{|c|}{7} & \multicolumn{2}{|r|}{8} & \multicolumn{2}{|r|}{9} & \multicolumn{2}{|c|}{ I0 } \\
\hline & Act. & Expd. & Act. & Expd. & Act. & Expd. & Act. & Expd. & Act. & Expd. & Act. & Expd. \\
\hline $\begin{array}{r}0 \\
1 \\
2 \\
3 \\
4 \\
5 \\
6 \\
7 \\
8 \\
9 \\
10\end{array}$ & $\begin{array}{r}\text { I I } \\
\text { I } 2 \\
\text { I0 } \\
4 \\
\text { O } \\
\text { O }\end{array}$ & $\begin{array}{r}8.71 \\
14.61 \\
9.80 \\
3.29 \\
0.55 \\
0.04\end{array}$ & $\begin{array}{r}12 \\
24 \\
19 \\
9 \\
4 \\
1 \\
0\end{array}$ & $\begin{array}{r}12 \cdot 16 \\
24 \cdot 4^{8} \\
20 \cdot 53 \\
9 \cdot 18 \\
2 \cdot 31 \\
0 \cdot 31 \\
0 \cdot 02\end{array}$ & $\begin{array}{r}24 \\
46 \\
44 \\
38 \\
17 \\
3 \\
0 \\
0\end{array}$ & $\begin{array}{r}22.70 \\
53.32 \\
53.65 \\
30.00 \\
10.06 \\
2.03 \\
0.23 \\
0.01\end{array}$ & $\begin{array}{r}19 \\
79 \\
6 \text { I } \\
49 \\
17 \\
5 \\
1 \\
0 \\
0\end{array}$ & $\begin{array}{c}22 \cdot 83 \\
61 \cdot 28 \\
71 \cdot 95 \\
48 \cdot 27 \\
20 \cdot 04 \\
5 \cdot 43 \\
0 \cdot 91 \\
0 \cdot 09 \\
\ldots\end{array}$ & $\begin{array}{r}\text { I I } \\
27 \\
36 \\
37 \\
16 \\
3 \\
0 \\
0 \\
0 \\
0\end{array}$ & $\begin{array}{r}9.62 \\
29.05 \\
38.98 \\
30.51 \\
15.35 \\
5.15 \\
1.15 \\
0.17 \\
0.01 \\
\ldots\end{array}$ & $\begin{array}{r}3 \\
18 \\
24 \\
11 \\
13 \\
1 \\
2 \\
0 \\
0 \\
0 \\
0\end{array}$ & $\begin{array}{c}3.88 \\
13.02 \\
19.65 \\
17.57 \\
10.32 \\
4.15 \\
1.16 \\
0.22 \\
0.03 \\
\ldots \\
\ldots\end{array}$ \\
\hline$\underset{p}{\chi^{2}}$ & & $8^{076}$ & & $\begin{array}{l}247 \\
7\end{array}$ & $\begin{array}{r}9 \\
0.05\end{array}$ & $\begin{array}{l}913 \\
j-0 \cdot 10\end{array}$ & & $\begin{array}{l}\cdot 024 \\
\cdot 15\end{array}$ & & $\begin{array}{l}847 \\
6\end{array}$ & & $\begin{array}{l}\text { I } 39 \\
2\end{array}$ \\
\hline
\end{tabular}

$\mathrm{SC}$, and in their selfed ovaries only one non-fusion ovule was seen amongst 2 I 22 fertilized ovules examined. Of the 63 SI clones, 62 gave homogeneous counts of non-fusion and normal ovules, totalling 2223 non-fusion (24.7I per cent.) and 6772 normal ovules in 242 selfed ovaries. The one remaining SI clone, ICS 90, gave counts which suggest a near-incidence of Ioo per cent. non-fusion ovules. Seven selfed ovaries gave:

\begin{tabular}{cc}
$\begin{array}{c}\text { Non-fusion } \\
\text { ovules }\end{array}$ & $\begin{array}{c}\text { Normal } \\
\text { ovules }\end{array}$ \\
27 & o \\
29 & I \\
25 & 2 \\
32 & 1 \\
25 & 2 \\
39 & 1 \\
40 & I \\
\hline 2 I 7 & -8
\end{tabular}

ICS go provides an example of yet a third type of SI tree; other examples will be mentioned later. 
TABLE 4

Ovule counts from self- and cross-pollinations on some genotypes investigated by Knight and Rogers

L.H. number refers to non-fusion ovules, R.H. number to normal ovules

\begin{tabular}{|c|c|c|c|c|c|c|c|c|c|c|}
\hline o & \multicolumn{2}{|c|}{$\begin{array}{l}S_{1 \cdot 2} \\
S_{1 \cdot 4}\end{array}$} & \multicolumn{2}{|c|}{$\mathrm{S}_{2 \cdot 3}$} & \multicolumn{2}{|c|}{$\mathrm{S}_{2 \cdot 3}$} & \multicolumn{2}{|c|}{$\mathrm{S}_{3 \cdot 4}$} & \multicolumn{2}{|c|}{$" S_{5.5} "$} \\
\hline $\begin{array}{l}S_{1 \cdot 2} \\
S_{1 \cdot 4}\end{array}$ & $\begin{array}{ll}10 \\
11 \\
11 \\
1 & 3 \\
1 & 3 \\
1 & 1 \\
1 & 1\end{array}$ & $\begin{array}{l}37 \\
33 \\
38 \\
35 \\
34 \\
40 \\
39\end{array}$ & $\begin{array}{l}0 \\
0\end{array}$ & $\begin{array}{l}43 \\
39\end{array}$ & $\begin{array}{l}0 \\
0\end{array}$ & $\begin{array}{l}47 \\
37\end{array}$ & $\begin{array}{l}0 \\
0\end{array}$ & $\begin{array}{l}49 \\
29\end{array}$ & 0 & 45 \\
\hline $\mathrm{S}_{2 \cdot 3}$ & $\begin{array}{l}0 \\
0\end{array}$ & $\begin{array}{l}4^{1} \\
5^{2}\end{array}$ & $\begin{array}{l}22 \\
24 \\
24 \\
24 \\
22 \\
24 \\
23 \\
25\end{array}$ & $\begin{array}{l}24 \\
19 \\
26 \\
25 \\
30 \\
23 \\
28 \\
19\end{array}$ & $\begin{array}{r}8 \\
6 \\
10 \\
10\end{array}$ & $\begin{array}{l}36 \\
17 \\
37 \\
41\end{array}$ & $\begin{array}{r}9 \\
9 \\
12\end{array}$ & $\begin{array}{l}31 \\
35 \\
3^{2}\end{array}$ & $\begin{array}{l}0 \\
0\end{array}$ & $\begin{array}{l}47 \\
47\end{array}$ \\
\hline $\mathrm{S}_{2 \cdot 5}$ & $\begin{array}{l}0 \\
0\end{array}$ & $\begin{array}{l}43 \\
46\end{array}$ & $\begin{array}{l}11 \\
19 \\
11\end{array}$ & $\begin{array}{l}33 \\
31 \\
44\end{array}$ & $\begin{array}{r}14 \\
12 \\
7 \\
5 \\
5 \\
12\end{array}$ & $\begin{array}{l}29 \\
35 \\
34 \\
24 \\
21 \\
3^{8}\end{array}$ & $\begin{array}{l}0 \\
0\end{array}$ & $\begin{array}{l}4^{8} \\
4^{8}\end{array}$ & $\begin{array}{l}0 \\
0\end{array}$ & $\begin{array}{l}54 \\
49\end{array}$ \\
\hline $\mathrm{S}_{3 \cdot 4}$ & $\begin{array}{l}0 \\
0\end{array}$ & $\begin{array}{l}49 \\
49\end{array}$ & 5 & $3^{2}$ & $\begin{array}{l}0 \\
0\end{array}$ & $\begin{array}{l}53 \\
39\end{array}$ & $\begin{array}{r}7 \\
14 \\
13 \\
15 \\
8 \\
14\end{array}$ & $\begin{array}{l}30 \\
34 \\
34 \\
33 \\
41 \\
34\end{array}$ & $\begin{array}{l}0 \\
0\end{array}$ & $\begin{array}{l}45 \\
52\end{array}$ \\
\hline$" S_{5.5} "$ & $\begin{array}{l}0 \\
0\end{array}$ & $\begin{array}{l}35 \\
37\end{array}$ & $\begin{array}{l}0 \\
0\end{array}$ & $\begin{array}{l}44 \\
40\end{array}$ & $\begin{array}{l}0 \\
0\end{array}$ & $\begin{array}{l}40 \\
47\end{array}$ & $\begin{array}{l}0 \\
0\end{array}$ & $\begin{array}{l}47 \\
47\end{array}$ & $\begin{array}{l}14 \\
10 \\
15 \\
12 \\
14 \\
11\end{array}$ & $\begin{array}{l}28 \\
30 \\
26 \\
34 \\
29 \\
31\end{array}$ \\
\hline 우 & & & & & & & & & & \\
\hline$S_{0.1}$ & $\begin{array}{l}22 \\
29 \\
29 \\
27\end{array}$ & $\begin{array}{l}30 \\
22 \\
24 \\
26\end{array}$ & II & 39 & $\begin{array}{l}0 \\
0\end{array}$ & $\begin{array}{l}53 \\
50\end{array}$ & & & & \\
\hline
\end{tabular}

$$
\mathrm{S}_{0}=\mathrm{S}_{1}>\mathrm{S}_{2}=\mathrm{S}_{3}>\mathrm{S}_{1}>\mathrm{S}_{8}
$$


Further confirmation of the association of non-fusion ovules with incompatibility, and of their absence with compatibility, was obtained when the author visited the West African Cocoa Research Institute and was able to make studies on some of the trees used by Knight and Rogers (1955) in their investigations. Counts from the selfand cross-pollinations made appear in table 4. All cases in which non-fusion ovules appeared were reported as incompatible by Knight and Rogers; and where these ovules were lacking, the pollination had been assessed by them as compatible.

$\mathrm{S}_{1.2}, \mathrm{~S}_{1.4}, \mathrm{~S}_{2.5}$ and $\mathrm{S}_{3.4}$-genotypes which combine dominant and recessive alleles - gave 206 non-fusion ovules $(24.26$ per cent.) amongst 849 fertilized ovules in I9 statistically homogeneous counts from selfed ovaries.

Selfed ovaries of $\mathrm{S}_{0.1}$ and $\mathrm{S}_{2.3}$ - genotypes with alleles independent in action-gave totals of 295 non-fusion ( 49.92 per cent.) and 296 normal ovules from 12 homogeneous counts.

Selfings on the $S_{5,5}$ genotype of Knight and Rogers showed 76 non-fusion and ${ }_{1} 78$ normal ovules in six homogeneous counts. The result strongly suggests that the trees examined belong to the common class of SI genotype giving 25 per cent. non-fusion ovules in selfed ovaries. $\mathrm{S}_{5.5}$ would more likely be $\mathrm{S}_{5 . x}$, where $\mathrm{S}_{x}$ is recessive to $\mathrm{S}_{5}$; this assumption in no way invalidates the hypothesis of Knight and Rogers.

Cross-pollinations between genotypes carrying the same dominant allele unfortunately were not made; but several pollinations between trees carrying alleles individual in action and others with one of these alleles as a dominant were carried out. Pollinations between $\mathrm{S}_{2.3}$ and $S_{2.5}, S_{2.3}$ and $S_{3.4}, S_{0.1}$ and $S_{1.2}$ gave I 1 I non-fusion $24 \cdot 30$ per cent.) and 408 normal ovules.

All other combinations of genotypes lacked non-fusion ovules in the cross-pollinated ovaries.

Fourteen of the many groups of progeny raised under the plantbreeding programme at the Imperial College of Tropical Agriculture were investigated for the incidence of self-compatibility and selfincompatibility, as judged by the non-appearance and appearance, respectively, of non-fusion ovules in selfed flowers. In several of the I 4 groups the reactions to back- and sib-pollinations were also studied. The progeny groups arise from the following types of parental cross(1) SC selfed: (2) $\mathrm{SC} \times \mathrm{SC}:(3) \mathrm{SC} \times \mathrm{SI}:(4) \mathrm{SI} \times \mathrm{SI}$. The parents involved in the crosses were:

\begin{tabular}{|c|c|c|}
\hline ICS I & SC & \\
\hline ICS 6 & $\mathrm{SC}$ & Trinitario clones \\
\hline ICS $_{44}$ & SC & \\
\hline ICS 45 & SC & Clone of strong Central-American criollo affinities. \\
\hline ICS 60 & SI & $\begin{array}{l}\text { Clone of strong Central-American criollo affinities, giving } \\
25 \text { per cent. non-fusion ovules when selfed. }\end{array}$ \\
\hline ICS 16 & SI & $\begin{array}{l}\text { Trinitario clone, giving } 25 \text { per cent. non-fusion ovules in } \\
\text { selfs. }\end{array}$ \\
\hline
\end{tabular}


SCA 12 SI

SCA 6 SI
Amazon clone, giving 25 per cent. non-fusion ovules in selfs. Amazon clone, giving 50 per cent. non-fusion ovules in selfs.

Results obtained from the progenies are given below in much condensed form, and the deductions made appear in italics. The figures to the left and right of the stroke are, respectively, the number of non-fusion and the number of normal ovules counted.

(1) ICS I selfed (SC selfed)

(2) ICS $_{1} \times$ ICS 6 $(\mathrm{SC} \times \mathrm{SC})$

(3) ICS $_{\mathrm{I}} \times \mathrm{ICS}_{44}$ $(\mathrm{SC} \times \mathrm{SC})$

(4) ICS 45 selfed ( $\mathrm{SC}$ selfed)

(5) ICS $_{\text {I }} \times$ ICS $_{45}$ $(\mathrm{SC} \times \mathrm{SC})$

(6) ICS $_{45} \times$ ICS I $_{\text {I }}$ $(\mathrm{SC} \times \mathrm{SC})$

(7) ICS $_{1} \times \operatorname{ICS}_{16}$ $(\mathrm{SC} \times \mathrm{SI})$

(8) ICS $60 \times$ ICS I $(\mathrm{SI} \times \mathrm{SC})$

(9) ICS $60 \times$ ICS 45 $(\mathrm{SI} \times \mathrm{SC})$

(10) ICS $45 \times$ ICS 60 $(\mathrm{SC} \times \mathrm{SI})$

(II) ICS $_{1} \times \mathrm{SCA}_{12}$ $(\mathrm{SC} \times \mathrm{SI})$
Selfings on 18 progeny gave $10 / 1120$. All progeny $S C$.

Selfings on 18 progeny gave $2 / 1306$. All progeny $S C$.

Selfings on 17 progeny gave $0 / 95^{8}$. All progeny SC.

Selfings on 25 progeny gave o/955. All progeny SC.

Selfings on 40 progeny gave $1284 / 3994$. All progeny 25 per cent. non-fusion SI types. One progeny, cross-pollinated with 16 sibs, gave ${ }_{13} 8 / 384$. All progeny cross-incompatible.

Selfings on 25 progeny gave $345 / 1255$. All progeny 25 per cent. non-fusion SI types. Pollinations with ICS I gave o/60 I, with ICS 45 gave $0 / 402$. All progeny cross-compatible with both parents.

Selfings on 20 progeny examined. 13 gave $123 / 399$; 7 gave $0 / 272$. I I I ratio for 25 per cent. SI and SC progeny. Pollinated with ICS 16 , the 13 gave $1_{32} / 374$. SI progeny cross-incompatible with SI parent. Pollinated with ICS I, all progeny gave o/716. All progeny cross-compatible with $S C$ parent.

Selfings on 20 progeny examined. 12 gave $123 / 33^{8}$; 8 gave I/431. I: I ratio for 25 per cent. SI and SC progeny. Pollinated with ICS 6o, the 12 gave 106/341. SI progeny cross-incompatible with SI parent. Pollinated with ICS I, all progeny gave o/846. All progeny cross-compatible with $S C$ parent.

Selfings on 37 progeny examined. I4 gave $733 / 46 ; 16$ gave $189 / 649 ; 7$ gave $1 / 388$. $3: 3: 2$ ratio for near-roo per cent. SI, 25 per cent. SI and SC progeny. Pollinated with ICS 6o, the 14 gave $240 / 248$; the 16 gave $125 / 404$; the 7 gave 1/388. SI progeny cross-incompatible, the SC progeny crosscompatible with SI parent. Pollinated with ICS 45, all progeny gave 1/605. All progeny cross-compatible with $S C$ parent.

Selfings on 22 progeny examined. 7 gave $394 / 14 ; 12$ gave $143 / 503 ; 3$ gave $4 / 149.3: 3: 2$ ratio for near-10o per cent. SI, 25 per cent. SI and SC progeny. Pollinated with ICS 60, the 7 gave 103/119; the 12 gave $102 / 331$; the 3 gave o/99. SI progeny cross-incompatible, the SC progeny cross-compatible with SI parent. Pollinated with ICS 45, all progeny gave o/473. All progeny cross-compatible with $S C$ parent.

Selfings on 15 progeny examined. All gave 296/903. All progeny 25 per cent. SI types. Pollinated with SCA I2, 8 gave 185/475, 7 gave 5/568. Equal numbers of progeny crossincompatible and cross-compatible with SI parent. Intra-group) 
pollinations gave $180 / 494$; inter-group pollinations gave I/5I4. Pollinated with ICS I, all progeny gave o/642. All progeny cross-compatible with SC parent.

(12) ICS $1 \times$ SCA $6 \quad$ Selfings on 15 progeny examined. All gave 289/932. All $(\mathrm{SC} \times \mathrm{SI}) \quad$ progeny 25 per cent. SI types. Pollinated with SCA 6 , all gave 333/942. All progeny cross-incompatible with SI parent. Two intra-incompatible but inter-compatible groups were found. Intra-group pollinations gave $237 / 707$; intergroup pollinations gave $\mathrm{I} / 936$. Pollinated with ICS I, all progeny gave 2/598. All progeny cross-compatible with $S C$ parent.

(13) ICS $60 \times \operatorname{SCA} 12$ Selfings on 32 progeny examined. 5 gave $105 / 128 ; 27$ $(\mathrm{SI} \times \mathrm{SI})$

(14) ICS $60 \times$ SCA 6 gave $372 / 1074 . \quad 1: 3$ ratio for 50 per cent. SI and 25 per cent. SI types. Pollinated with ICS 6o, the 5 gave $49 / 132$; with SCA 12 pollen, they gave $43 / 152.50$ per cent. SI progeny cross-incompatible with both SI parents. The 27 fell into three classes according to their reaction with parental pollens. 12 gave $0 / 420$ with ICS 60, and 114/310 with SCA 12 ; I I gave Ioo/334 with ICS 6o, and o/406 with SCA I2; 4 gave $0 / 307$ with pollen from both parents. $I: I: I: I$ ratio for the four progeny types.

$(\mathrm{SI} \times \mathrm{SI})$

Selfings on 21 progeny examined. 9 gave $225 / 264 ; 12$ gave $169 / 462$. I $I$ ratio for $5^{\circ}$ per cent. SI and 25 per cent. SI types. Pollinated with ICS 6o, the 9 gave $91 / 300$; the 12 gave $1 / 505$. 50 per cent. SI progeny cross-incompatible, the 25 per cent. SI progeny cross-compatible with 25 per cent. SI parent. Pollinated with SCA 6, all progeny gave 191/626. All progeny cross-incompatible with 50 per cent. SI parent.

\section{Review of all counts mode}

A study of the results given above shows that self- and crosspollinations in cacao can be classified as (I) compatible: (2) leading to the incidence of 25 per cent. of non-fusion ovules: (3) leading to the incidence of 50 per cent. of non-fusion ovules: (4) leading to the incidence of nearly Ioo per cent. of non-fusion ovules. Counts from all four cases are summarised:

(I) Compatible matings. 819 ovaries from compatible selfings and crosses contained 67 non-fusion ovules and 29,457 normal. The non-fusion ovules were contained in only 38 of these ovaries, so that the majority of compatible matings are characterised by a ioo per cent. incidence of normal ovules.

(2) 25 per cent. non-fusion matings. In I I 73 self- and crosspollinations made there were II, I57 non-fusion ovules (24.7433 per cent.) and 33,934 normal ovules $(75 \cdot 2657$ per cent.). The weighted mean of the transformed data is $0.5393^{2} \pm 0.0055^{2}$ and the ratios corresponding to the 5 per cent. fiducial limits are I :2.88 and I : 3.0 I. The I I 73 components of contingency- $\chi^{2}$, when grouped according to magnitude, give reasonably good fit with expectation (table 5); the data are statistically homogeneous. Of these I I 73 ovaries, 645 contained numbers of fertilized ovules ranging from 35 to 44 . In each of the ten groups, characterised by the possession of $35,36,37 \ldots 44$ 
ovules, the frequency of occurrence of from zero to 20 non-fusion ovules was scored; these frequencies showed good fit, in all ten arrays, with theoretical frequencies derived from the expansion of $(0.247433+$ $0 \cdot 752657)^{n}$, where $n$ is the number of fertilized ovules in the ovary. The encounter of non-fusion elements may therefore be regarded as a randomly controlled process.

(3) 50 per cent. non-fusion matings. In 57 ovaries which showed an approximation to a 50 per cent. incidence of non-fusion ovules there were collectively 1075 non-fusion and $I_{15} I$ normal ovules. The counts are homogeneous.

TABLE 5

Frequency distribution of contingency- $x^{2}$ values (calculated from ratio, 11157:33934) for the II73 counts from ovaries showing an incidence of 25 per cent. non-fusion ovules

\begin{tabular}{|c|c|c|c|}
\hline $\begin{array}{l}\chi^{2} \text { range } \\
\text { for I d.f. }\end{array}$ & $\begin{array}{c}\text { Actual } \\
\text { frequency }\end{array}$ & $\begin{array}{c}\text { Expected } \\
\text { frequency }\end{array}$ & $\chi^{2}$ \\
\hline 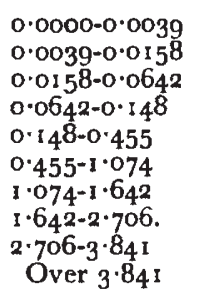 & $\begin{array}{r}56 \\
68 \\
118 \\
119 \\
251 \\
239 \\
111 \\
118 \\
39 \\
54\end{array}$ & $\begin{array}{l}58 \cdot 65 \\
58 \cdot 65 \\
117 \cdot 3 \\
117 \cdot 3 \\
234 \cdot 6 \\
234 \cdot 6 \\
117 \cdot 3 \\
117.3 \\
58 \cdot 65 \\
58 \cdot 65\end{array}$ & $\begin{array}{l}0.1197 \\
1.4906 \\
0.0042 \\
0.0246 \\
1 \cdot 1465 \\
0.0825 \\
0.3384 \\
0.0042 \\
6.5835 \\
0.3687\end{array}$ \\
\hline Totals & I1 73 & $1173^{\circ} 0$ & $\begin{array}{c}10 \cdot 1629(9 \mathrm{~d} . \mathrm{f}) . \\
p=0.34\end{array}$ \\
\hline
\end{tabular}

(4) Ioo per cent. non-fusion matings. In 52 pollinations there appeared 1564 non-fusion ovules and 93 normal. Seventeen of the ovaries entirely lacked normal ovules. Contingency- $\chi^{2}{ }_{(51)}$ is 127.635 ( $p$ very low), so that the counts are significantly heterogeneous.

All three groups in which non-fusion ovules appear show deficits in the numbers counted if it is assumed that the ratios of non-fusion to normal ovules should exactly be I : 3 , I : I and I : o, respectively. In the case of the 25 per cent. non-fusion class of mating, the deficit is of the order of I per cent.; in the 50 per cent. class it exceeds 3 per cent.; and in the 100 per cent. non-fusion class the deficit approaches 6 per cent. Some significance may attach to the fact that as the ratio of non-fusion to normal ovules becomes larger, so the deficit of non-fusion ovules is more marked.

In order to discover whether or not, in incompatible pollinations, a regular proportion of pollen tubes fails to reach the embryo-sacs and effect fertilization, a series of pollinations was made in the laboratory. Small, profusely flowering branches of the SI clones, SCA 6 and SCA I2, were removed from the trees and brought into the laboratory where, under a low-power binocular microscope, 
counted numbers of pollen grains were applied to the stigmas of flowers carefully checked for absence of pre-pollination. All other flowers were then stripped off the branches, which were placed in water under bell-jars. Three days later the pollinated flowers were removed and the ovaries fixed and sectioned. With the number of pollen grains applied ranging from six to $4 \mathrm{I}$, the ovary sections clearly revealed that in all cases nearly 100 per cent. of the grains had effected fertilization. No detectable morphological or cytological differences, indeed, were ever observed amongst the grains in samples of pollen taken from the anthers of SI (and SC) trees. The grains are bi-nucleate at anthesis.

\section{GENETICAL INTERPRETATION}

The central facts which have emerged from the investigations reported in this paper are ( 1 ) that incompatible matings in Theobroma cacao, which lead to flower abscission, are characterised by the presence of 25,50 or near-1oo per cent. of non-fusion ovules in the ovary: (2) that compatible matings typically lack these non-fusion ovules.

Statistical analyses have consistently shown, where replication permits, that between-tree and within-tree variances of the ratio of non-fusion to normal ovules in incompatible matings are of very similar magnitudes. The amount of spread in the ovule ratio, from ovary to ovary, has been shown by contingency- $\chi^{2}$ analyses to be in conformity with the demands of random sampling. The ovule counts obtained clearly demonstrate the operation of a factor, or factors, constant in effect and subject only to sampling error. Other analyses have shown unequivocally that the encounter between gametes which fail to fuse is a strictly random process. All pollen tubes in incompatible matings have been shown to grow equally well in the style, and no evidence has been obtained to suggest that the penetration of embryo-sacs by pollen tubes bearing incompatible gametes is in any way selectively controlled.

The results obtained from the investigations on materials actually used by Knight and Rogers are of great interest. The patterns of abscission and retention of pollinated flowers obtained by these authors amongst their three groups of progeny led them to postulate the existence of sporophytic control of the cacao incompatibility reaction on both male and female sides; a single locus, with multiple alleles showing dominance and independence relationships $\left(S_{1}>S_{2}=\right.$ $\mathrm{S}_{3}>\mathrm{S}_{4}>\mathrm{S}_{5}$ ), appeared to be involved.

In the studies reported here, the commonest manifestation of the incompatibility of self- and cross-pollinations is the appearance of 25 per cent. non-fusion ovules in the ovary. Evidently, then, the gametes involved in the fertilization processes are not all physiologically identical in their fusion and non-fusion reactions, for otherwise an incidence of 100 per cent. non-fusion ovules would be the rule in these (and, indeed, in all) incompatible matings in cacao. The 
reaction of male and female gametes towards each other would appear to depend solely on their individual haploid constitutions; such control of the incompatibility reaction would seem to conflict with the sporophytic control postulated by Knight and Rogers. The following considerations reveal the fact, however, that in certain circumstances there two hypotheses need not be in conflict. The presence of non-fusion ovules in the cacao ovary results in abscission of the flower. If the likely assumption is made that male and female gametes carrying the same allele are those that cannot fuse, then genotypes with a common $\mathrm{S}$ allele would be cross-incompatible; and cross-compatibility would result only when all four $\mathrm{S}$ alleles of two parent genotypes were different. All progeny of the cross would be incompatible with both parents, and any one of the four progeny groups would be compatible with only one other. Genotypes homozygous for $\mathrm{S}$ alleles, furthermore, could not exist. Haploid control of gamete reaction towards fusion, and fall of the flower once nonfusion within a proportion of the ovules had occurred, would therefore be indistinguishable in effect from sporophytic control of incompatibility (through pollen tube and style interaction) on male and female sides with independence of $\mathrm{S}$ allele action (Bateman, 1952Scheme F).

Though haploid control of syngamy thus exactly simulates sporophytic style-and-pollen-tube control when S allele action is independent, it fails to provide an explanation of the facts that in cacao ( 1 ) progeny are usually compatible with one or other parent-sometimes with both-in crosses between SI parents: (2) homozygotes can be identified. These circumstances require dominance relationships to exist between the alleles, as Knight and Rogers postulated. We are then left with the necessity of explaining why the gametes in a genotype which carries one $\mathrm{S}$ allele dominant to the other are not all phenotypically identical in their fusion behaviour, since only 25 per cent. non-fusion ovules, and not roo per cent., are found in selfed ovaries of such genotypes.

The cross-relationships of $\mathrm{S}_{2.3}, \mathrm{~S}_{2.5}$ and $\mathrm{S}_{1.2}$ clearly indicate that non-fusing gametes are those carrying the dominant allele. Crosses between the first two, in either direction, give 25 per cent. non-fusion ovules in the ovary (table 4). In the cross, one-quarter of the gametic encounters will be between $S_{2}$ gametes, and three-quarters between gametes carrying unlike alleles. It seems therefore logical to suppose that non-fusion ovules result from the delivery of $S_{2}$ male gametes into embryo-sacs carrying $\mathrm{S}_{2}$ female nuclei-in $\mathrm{S}_{2.3}$ and $\mathrm{S}_{2.5}$ the $\mathrm{S}_{2}$ gametes, male and female, are "activated" against fusion. $S_{2.3}$, according to Knight and Rogers, carries alleles independent in action, whilst $\cdot S_{2.5}$ has $S_{2}$ dominant to $S_{5}$. The selfing of $S_{2.5}$ also gives a 25 per cent. incidence of non-fusion ovules, so the conclusion is inescapable that, as the $\mathrm{S}_{2}$ gametes of this genotype are activated against fusion, the $\mathrm{S}_{5}$ gametes cannot be. The concept of the dominant-allele 
gametes being the only ones activated is strengthened by the compatible reaction of the $S_{2.5}$ genotype with $S_{1.2}$. Non-fusion ovules are lacking in the reciprocal crosses (table 4 ), so that $S_{2}$ gametes from $S_{2.5}$ and $S_{1.2}$ can readily fuse with each other; we deduce, therefore, that $S_{2}$ gametes derived from $S_{1.2}$ are not activated. It follows, then, that the incidence of 25 per cent. non-fusion ovules in the selfing of $S_{1.2}$ can arise only from encounters between $\mathrm{S}_{1}$ gametes.

The restriction of non-fusion to gametes carrying the same allele as dominants must mean that the $\mathrm{S}$ locus has a two-step action, one before meiosis to establish the dominance of one allele over another, the second after meiosis to give the genotypic reaction between gametes. One way in which this could be achieved would be by some form of interaction between haploid gamete nucleus and "diploid" cytoplasm. As a working hypothesis, it is suggested that the cytoplasm associated with the male and female gametes contains an incompatibility precursor substance which has been endowed by the dominant $\mathrm{S}$ allele, pre-meiotically, with some property such that only gametes receiving the dominant allele at meiosis and segregation can develop the precursor to give full and highly specific non-fusion properties to such gametes. Where independent $\mathrm{S}$ alleles occur in a genotype, the pre-meiotic action of the alleles would be such that the gametic cytoplasm contains precursors peculiar to each allele; after meiosis, both sets of gamete nuclei develop their own precursor to full non-fusion properties. These genotypes should, and do, show an incidence of 50 per cent. non-fusion ovules in selfed ovaries; they should, moreover, give 25 per cent. non-fusion ovules when crosspollinated with genotypes which carry one of the independent alleles as a dominant $\left(\mathrm{S}_{2.3} \times \mathrm{S}_{2.5}\right.$ and $\mathrm{S}_{2.3} \times \mathrm{S}_{3.4}$ in table 4$)$.

The hypothesis that gametes carrying the same dominant allele are those that do not fuse would require SI genotypes homozygous for an $\mathrm{S}$ allele to show Ioo per cent. fusion failure. Such types have been mentioned (viz, ICS 90 and progenies of reciprocal crosses between ICS 45 and ICS 6o). The observed frequent failure of self-pollinations to give non-fusion in every fertilized ovule must mean that these failures represent a level of activation insufficient to prevent syngamy.

The reason why only three types of SI cacao tree have been found is now clear. The two S alleles in any tree can be either ( $I$ ) both of. different dominance status: (2) both different but independent in action: (3) both alike. The first case provides for the incidence of 25 per cent. non-fusion ovules in selfed ovaries, the second will represent the $5^{\circ}$ per cent. non-fusion type, and the third will represent the Ioo per cent. non-fusion SI tree. Where $n$ alleles exist in a cacao population, and a fraction, $p$, of them are individual in action, the numbers of possible allelic combinations are

$\frac{1}{2} n^{2}\left(\mathrm{I}-p^{2}\right)-\frac{1}{2} n(\mathrm{I}-p)$ for the 25 per cent. non-fusion SI class $\frac{1}{2} n p(n p-1)$ for the 50 per cent. non-fusion SI class

and $n-I$ for the 100 per cent. non-fusion SI class. 
In general, the first group will be the largest numerically since the expression contains terms in $n^{2}$. Only if $p$ exceeds the limiting value of $\sqrt{ } 2 / 2$ will it be possible for the second and first groups to be equal. Experience shows that the 25 per cent. non-fusion SI type is the most commonly encountered one.

Selfings and crosses involving SC Trinitario clones as parents have been shown to yield only SC progeny (ICS I selfed, ICS I $\times$ ICS 6 , ICS I $\times$ ICS 44). The true-breeding properties of this material suggest that it is homozygous for the compatibility factor or factors, and rule out the suggestion made by Pandey (1960) that self-compatibility in cacao is the result of competitive allelic interaction. ICS 45 , a clone of strong Central-American criollo affinities, is also a true-breeding SC type. The cross between ICS I and ICS 45 , however, gives progeny which are all 25 per cent. non-fusion SI types, and they appear, moreover, to be mutually cross-incompatible. One of the parents must therefore be homozygous for an active $S$ allele, the activity of which must in some way be suppressed, with restoration in the progeny. It seems reasonably certain, then, that ICS I and ICS 45 are homozygous at two (or more) different loci which show complementary action to produce the SI condition in the offspring. The inter-relationships of ICS I, ICS 45 and ICS 60 disclose the fact that it is ICS 45 which is homozygous for an active $S$ allele. ICS 60 is a 25 per cent. non-fusion SI clone which, with ICS I, gives progeny which are SI and SC in approximately equal numbers. ICS 60 must therefore be heterozygous for (I) an active $S$ allele: (2) the compatibility factor of ICS I, which must be recessive to the active S allele. When ICS 60 is hybridised with ICS 45 , a proportion of the progeny are near-Ioo per cent. non-fusion SI genotypes, and these, being homozygous, must have received the same $\mathrm{S}$ allele from both parents. ICS 45 is therefore the SC genotype which is homozygous for an active $\mathrm{S}$ allele, rendered ineffective by another locus or other loci. The fact that the $\mathrm{S}$ allele is dominant to the compatibility factor in ICS 60 supports the suggestion that the latter is an inactive amorph of the $\mathrm{S}$ series. If so, then ICS I would be homozygous for the amorph (which will be denoted by $\mathrm{S}_{f}$ ) and the accessory locus or loci will be active in it in order to impart self-incompatibility to its progeny with ICS 45 . These same inter-relationships, furthermore, require two other loci, with the $\mathrm{S}$ locus, to be concerned in the establishment of the SI and SC conditions. Appeal to only one other locus has been found incapable of explaining the segregations obtained in crosses between the three parents. These two accessory loci, showing simple dominance and recessivity, have been called $\mathrm{A}$ and B; it is supposed that both must be at least heterozygous for the dominant alleles before any active alleles at the $\mathrm{S}$ locus can impart an incompatibility reaction to the gametes. ICS 45 must be either

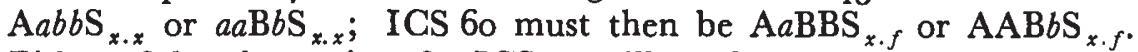
Either of the alternatives for ICS 45 will produce all SI offspring with 
ICS I, thought to be $\mathrm{AABBS}_{f . f}$, and either alternative for ICS 60 will produce equal numbers of SC and SI progeny with ICS I.

The results obtained from the studies of progenies of eight parent clones suggest that the parents have the following genotypes:

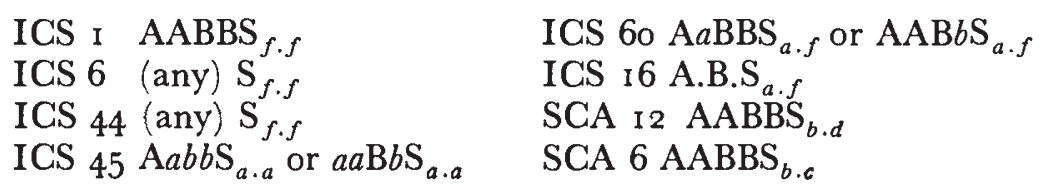

where $\mathrm{S}_{a}=\mathrm{S}_{b}=\mathrm{S}_{c}>\mathrm{S}_{d}>\mathrm{S}_{f}$.

The need for the A and B loci, to act in concert with the $\mathrm{S}$ locus, and the marked specificity of the $S$ alleles would support the suggestion made above that the $\mathrm{S}$ locus acts upon some substance or substances to give modified substances which are highly specific to the individual $\mathrm{S}$ alleles. It is tempting to suppose that the $\mathrm{A}$ and $\mathrm{B}$ loci produce an "incompatibility-substance precursor" on which the $\mathrm{S}$ alleles act. If either, or both, of them should be inactive, the $\mathrm{S}$ locus would have no precursor on which to act; in this circumstance the tree is SC (e.g., ICS 45). If the $\mathrm{S}$ allele is an inactive amorph in the homozygous condition, then the precursor formed by the A and B loci cannot undergo modification to specific characteristics, and the tree will again be SC (e.g., ICS I). Both SC genotypes will produce gametes compatible with those of any other cacao genotype, a fact which has, indeed, been known for a long time.

The hypothesis put forward by Bennett and Cope (1959), in explanation of non-fusion of nuclei, has the merit of accounting for the not infrequent dissociation of polar nuclei in embryo-sacs which have received incompatible male gametes. When fusion takes place between polar nuclei and a male gamete compatible with them, the male nucleus invariably makes contact with the paired polar nuclei at some point on their interface; the three nuclei come, in fact, to lie in mutual contact, the male nucleus then insinuating itself between the polar nuclei, assuming a more and more fusiform shape as it penetrates. The contents of the male nucleus eventually mingle with the nucleoplasm of one polar nucleus, so that the primary endosperm body presents the appearance of two nuclei, back to back, one with two nucleoli and the other with one (plate, fig. 4). This fusion behaviour can be likened to the coalescence of oil droplets in an unstable oil/water emulsion, brought about by the tendency of the droplets to have maximal shared surface areas. The male gamete, originally spherical (as is an isolated droplet), positions itself with respect to the polar nuclei in such a manner that its entire surface area becomes shared with these two nuclei in the process of fusion.

In the case of non-fusing nuclei, entirely different interfacial conditions must exist, and the system can be likened to a stable oil/ water emulsion wherein each droplet is stabilised by the formation of a monolayer of some protective substance over its surface, so that 
direct oil-to-oil contact between droplets is prevented. Coalescence, in these circumstances, does not take place. The restriction of nonfusion to nuclei carrying the same dominant allele implies that the stabilization process is associated only with them. We can suppose that there is present in the cytoplasm of the male gamete carrying the dominant allele a strongly adsorbed protective substance specific to that allele; the female nuclei carrying the dominant allele have specific reactive sites on the nuclear membrane. If the dominant allele carried by the male and female nuclei is identical, the protective substance, diffusing from the male cytoplasm, interacts with the specific sites on the surface of the female nuclear membrane, leading to stabilization-a result which can cause the polar nuclei to separate. Female nuclei carrying the recessive allele are without specific reactive sites, for these nuclei will fuse with any male nucleus; and male gametes carrying the recessive allele can have no protective substance associated with them, because fusion between them and any female nuclei can always take place. The association of the protective substance with the male nucleus and cytoplasm rather than with the female nuclei seems more likely, since female nuclei show no tendency towards separation until the male gamete comes into their vicinity. Female egg and synergid nuclei, moreover, have been seen to fuse in the absence of fertilization. In view of the pronounced specificity of the interaction between the protective substance and sites present on the female nuclear membrane, the substance would seem likely to be proteinaceous.

\section{DISCUSSION}

The incompatibility mechanism of cacao and the genetic system controlling it are unlike those of any other flowering plant so far recorded. Fertilization processes are identical in compatible and incompatible matings right up to the time that male and female gametes come into contact in the embryo-sac. The genetic system has some aspects of both major systems controlling incompatibility in other angiosperms; the gametophytic and sporophytic systems differ fundamentally in the time of $\mathrm{S}$ gene action during microsporogenesis (but are alike in having sporophytic control on the female side). In cases of gametophytic control, the $\mathrm{S}$ locus exerts its influence after tetrad formation: in cases of sporophytic control, before tetrad formation. With cacao, an explanation of the events observed and the results obtained is reached by assuming that the $\mathrm{S}$ locus acts both before and after meiosis, on the female side as well as on the male.

It is interesting to speculate on the reason for the combination, in the cacao system, of aspects of gametophytic and sporophytic control. If the non-fusion reaction between male and female gametes depended solely upon identity of S allele content, and if the presence of ovules in the ovary wherein syngamy failed was sufficient to cause 
flower abscission, we should have-as was mentioned in the previous section-a breeding system indistinguishable from that produced by sporophytic pollen-tube-and-style control with independence of $\mathrm{S}$ allele action. Bateman (1952) has pointed out the great disadvantage of the latter system; low cross-fertility exists unless the $\mathrm{S}$ alleles are numerous. Selection for large numbers of alleles and/or for the development of dominance relationships between them would be strong; but dominance relationships between $\mathrm{S}$ alleles require premeiotic action of the $\mathrm{S}$ locus for their development, so that an incompatibility system which was gametophytically controlled on male and female sides would be likely to evolve selectively into one in which the gametophytic reaction between gametes was supplemented by the effects of pre-meiotic $\mathrm{S}$ gene action.

Some authors have claimed that the incompatibility system in cacao is primitive for the reason that discrimination against the union of like gametes takes place in the embryo-sac and not in the style, a condition which leads to the loss of female gametes once the ovary has been incompatibly pollinated. Mather (1944) and Darlington and Mather (1950) go so far as to say that because no stylar sieve operates to conserve female gametes, the term "incompatibility" can scarcely be applied to the cacao mechanism. Knight and Rogers (1955) state, "In cacao, there is no inhibition in the style.... This may be a primitive condition since it leads . . . to the loss of ovules ". The primitiveness or otherwise of the cacao incompatibility system cannot be discussed, however, without reference to the biology of the tree. One striking characteristic of the species is the large number of flowers produced and the small number of mature fruits which develop from them. Cope (1939a) investigated the flowering intensity, setting and physiological wilt-loss of young fruits, and final crop in a population of young seedling cacao trees. The SC members of the population produced an average of 3358 flowers per tree in 12 months, but from this large number only 7.5 pods per tree came to maturity. Making the reasonable assumption that the ovary contains 40 ovules and the mature fruit contains $3^{6}$ seeds, we see at once that the chance of an ovule on the SC tree becoming a mature seed is about one in 500. The SI trees of the population, in the same period, bore an average of 5207 flowers per tree and yielded an average of 4.4 pods per tree; on these SI trees, the chance of an ovule becoming a ripe seed is one in 1300 . These figures certainly demonstrate the probability of successful mating in the SI tree to be smaller than in the SC tree, but even in the latter, where no incompatibility barriers exist, 499 out of every 500 ovules are lost as a result of a very low level of natural pollination and, in the successfully pollinated flowers, subsequent loss of zygotes from physiological wilt. Good evidence exists to show that if pollination were more effective than it actually is, the wilt of developing fruits would be more pronounced, thereby tending to keep the ratio of viable zygotes to female gametes 
constant. In the circumstance of extremely heavy loss of female gametes from causes other than incompatibility, the assertion of Knight and Rogers (I955) that "to achieve incompatibility in cacao there is an enormous loss of flowers" cannot be justified. The need for a stylar sieve in cacao scarcely arises, for no advantage would be gained by leaving a flower open to a second (and perhaps compatible) pollination when so many still-unpollinated flowers are available on the tree. Conservation of female gametes until such time as compatible male gametes are delivered into the embryo-sac would likely be essential for the survival of short-lived species which bear relatively few flowers in the course of the individual's lifetimebut such is not the case for cacao. There are no good grounds for regarding the incompatibility mechanism in cacao as primitive merely on the score of loss of female gametes, the great majority of which, in any case, is destined never to be mated.

Why incompatibly pollinated cacao flowers absciss is not known. In most ovaries of flowers that fall because of incompatible pollination there are present some 75 per cent. of the fertilized ovules which appear to have full potentialities for further development (fertilized egg and polar nuclei), yet mature pods can frequently be found which contain seeds numbering less than half the available ovules. The abscission of incompatibly pollinated flowers would seem, therefore, to be directly connected with the presence of non-fusion ovules. The nucellar cells lining the embryo-sac in non-fusion ovules often show signs of cellular breakdown. Possibly, then, the products of degeneration from the non-fusion ovules interfere with the development of the normal ovules, mitotic activity in the nucellus and integuments of which is very low at the time of abscission.

\section{SUMMARY}

I. Numerous investigations have shown that in incompatible matings in Theobroma cacao $\mathrm{L}$. there is always present in the ovary a proportion of ovules wherein syngamy has not taken place (" nonfusion ovules") and-usually-another proportion in which fusion is normal (" normal ovules").

2. In compatible matings, non-fusion ovules are usually entirely absent, though they may sporadically be encountered.

3. Counts of non-fusion and normal ovules in replicated incompatible pollinations have been statistically treated. Within- and between-tree variations in the ratio of non-fusion to normal ovules are very similar, and the spread in the ratio, from ovary to ovary, is no larger or smaller than random sampling theory would require. The frequency distribution of the number of non-fusion ovules found in groups defined by the loculus points to the encounter between gametes unable to fuse in the embryo-sac as being a purely random process. 

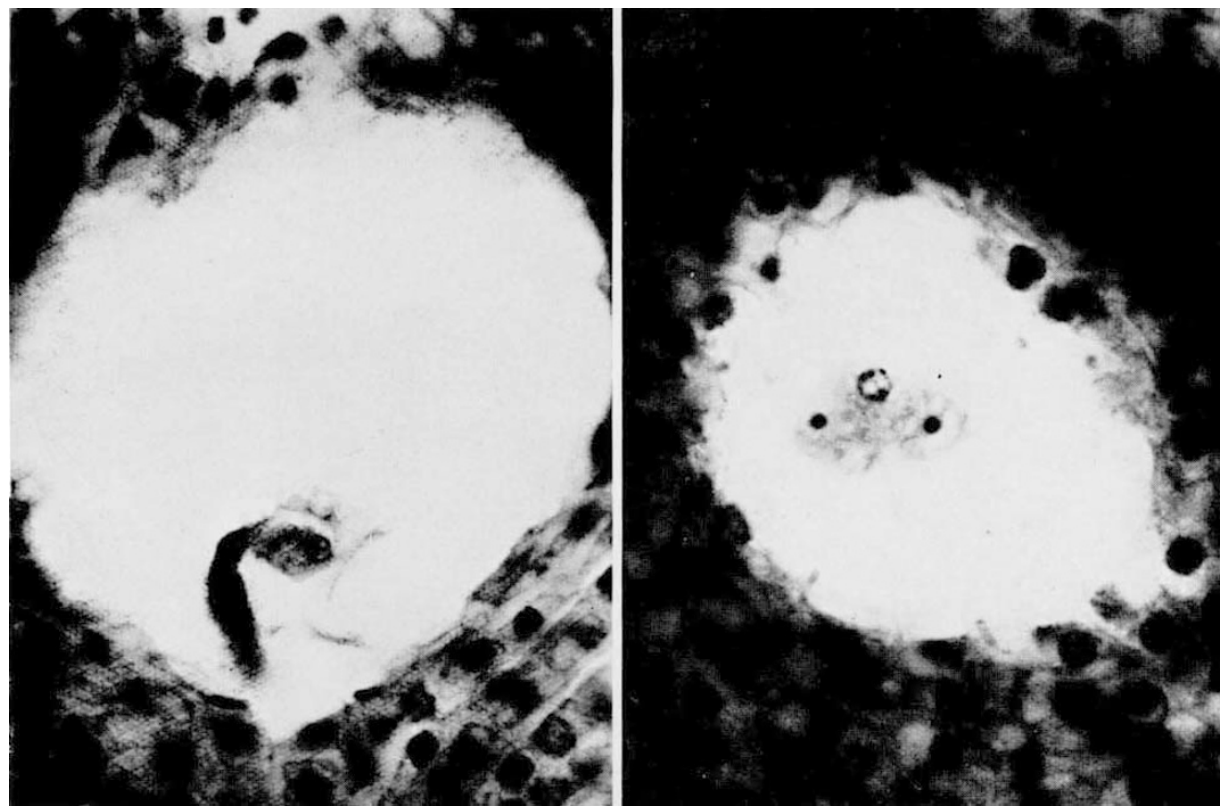

FIGs. I and 2.-Embryo-sac of non-fusion ovule, 72 hours after incompatible pollination. The unfused male and egg nuclei lie in contact to the right of the darkly-stained collapsed synergid in fig. I. The second male gemete lies in contact with the polar nuclei in fig. 2 . $\times 720$.
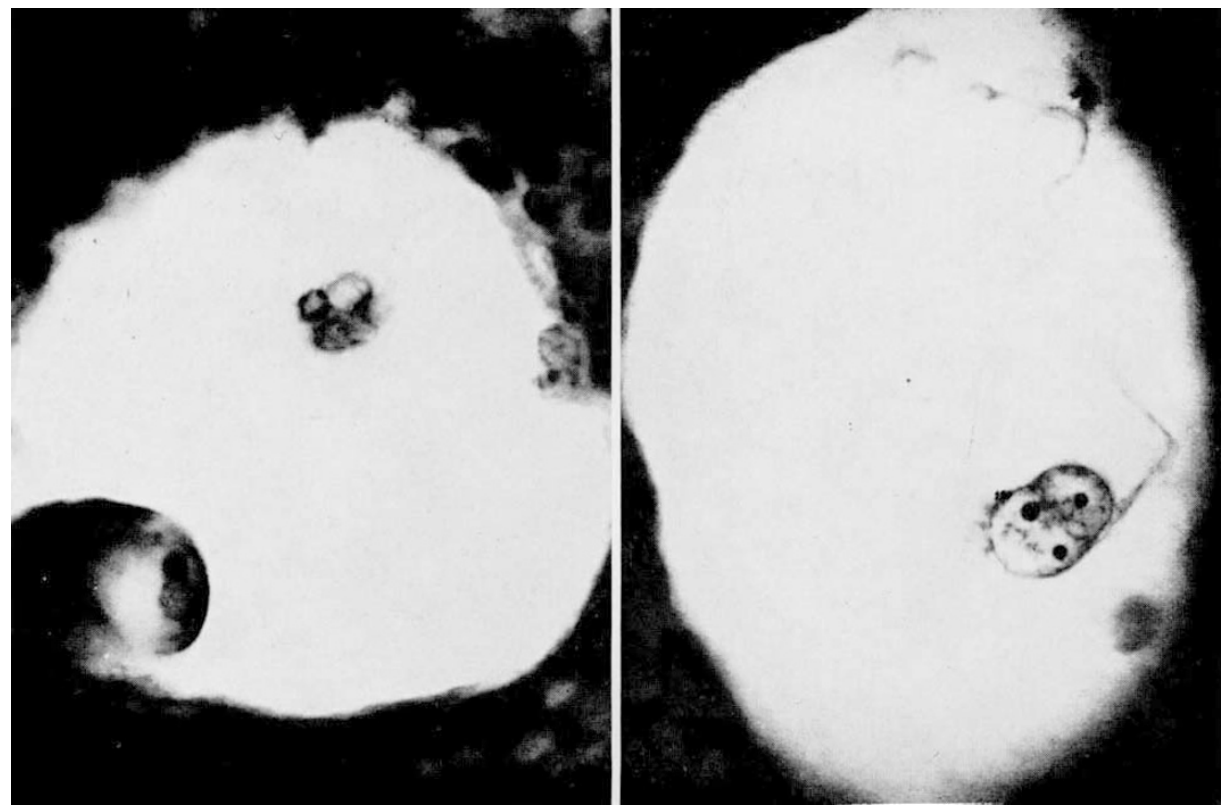

Fig. 3.-Embryo-sac of non-fusion ovule, 72 hours after incompatible pollination. The unfused male and egg nuclei can be seen in the bottom L.H. corner. The second male gamete and one polar nucleus lie above the centre of the sac, whilst the second polar nucleus lics against the R.H. side of it. $\times 720$.

FIg. 4.-Embryo-sac of normal ovule, 72 hours after compatible pollination. The primary endosperm body consists of two nuclei, back to back, one with two nucleoli, the other with one nucleolus. $\times 720$. 
4. Three types of SI cacao tree can be recognised by their giving, respectively, 25, 50 and near-1 100 per cent. proportions of non-fusion ovules in selfed ovaries.

5. The experimental results are consistent with the hypothesis that gametes carrying the same allele as a dominant cannot fuse. In genotypes with alleles of independent action both sets of gametes are activated against fusion with others of like haploid genotype. The $\mathrm{S}$ locus appears to have its action both before and after meiosis. Two other loci, called $\mathrm{A}$ and $\mathrm{B}$, are concerned in the incompatibility reaction; the role of these loci, possibly, is to produce non-specific precursors to which the $\mathrm{S}$ alleles impart their specificities. Genotypes homozygous for inactive alleles at one or more of the A, B and S loci will be selfcompatible.

\section{REFERENCES}

BAteman, A. J: 1952. Self-incompatibility in angiosperms. I-Theory. Heredity, $6,285-310$.

BENNETT, M. C., AND COPE, F. W. 1959. Nuclear fusion and non-fusion in Theobroma cacao L. Nature, London, $183,1540$.

COPE, F. W. 1939a. Some factors controlling the yield of young cacao II. 8th Ann. Rep. on Cacao Res. (1938), Trinidad, 4-15.

COPE, F. W. 1939b. Studies in the mechanism of self-incompatibility in cacao I. 8th Ann. Rep. on Cacao Res. (1938), Trinidad, 20-21.

COPE, F. W. 1940. Studies in the mechanism of self-incompatibility in cacao II. gth Ann. Rep. on Cacao Res. (1939), Trinidad, 19-23.

darlington, c. D., AND mather, K. 1950. Genes, Plants and People. Allen \& Unwin, London.

Johansen, D. A. 1940. Plant Microtechnique. McGraw-Hill, New York and London. KNIGHT, R., AND ROGERS, H. H. 1953. Sterility in Theobroma cacao L. Nature, London, $x 72, \mathrm{x} 64$.

KNIGHT, R., AND ROGERS, H. H. 1955. Incompatibility in Theobroma cacao. Heredity, 9, 69-77.

marshall, J. 1933. Fertility in Cacao. MSS. dissertation in Library of Imperial College of Tropical Agriculture, Trinidad.

mather, K. 1943. Specific differences in Petunia. I-Incompatibility. 7. Genet., $45,215-235$.

MATHER, K. 1944. Genetical control of incompatibility in angiosperms and fungi. Nature, London, 153, 392-394.

MÜNTZING, A. 1947. Some observations on the pollination and fruit setting in Ecuador cacao. Hereditas, 33, 397-404.

NAUNDORF, G., AND villamil, G. F. 1950 . Contribución al estudio de la fisiología del cacao (Theobroma cacao L.), II. Palmira Estac. Agr. Notas Agronómicas (Palmira, Colombia), 3, 87-90.

OSTENDORF, F. w. 1948. Fertility in cacao. Chron. Nat., 104, 101-105.

PANDEY, K. K. 1960. Incompatibility system in Theobroma cacao. Am. Nat., 94, 379-381.

posnette, A. F. 1937. Incompatibility and Pollination in Cacao. MSS, dissertation in Library of Imperial College of Tropical Agriculture, Trinidad.

PosNette, A. F. 1945. Incompatibility in Amazon cacao. Trop. Agriculture (Trin.), 22, $184-187$.

pound, F. J. 1932. Studies in fruitfulness in cacao, I and II. Ist Ann. Rep. on Cacao Res. (1931), Trinidad, 24-28.

pound, F. J. 1938. Cacao and Witchbroom Disease of South America. Yuille's Printerie, Trinidad. 
RANDOLPH, L. F. 1935. A new fixing fluid and a revised schedule for the paraffin method in plant cytology. Stain Tech., 1o, 95-96.

voelcker, o. J. 1937. Self-incompatibility in cacao, I. 6th Ann. Rep. on Cacao Res. (1936), Trinidad, $2-5$.

voelcker, o. J. 1938. Self-incompatibility in cacao, II. 7th Ann. Rep. on Cacao Res. (1937), Trinidad, 2-5.

yates, F. 1953. Sampling Methods for Censuses and Surveys. 2nd edition. Griffin \& Co., London. 